\title{
Early Rehabilitation Nursing for Stroke Patients with Hemiplegia in Neurology Department
}

\author{
Guixia Jiang* \\ Lianshui County People's Hospital, Lianshui County, Huai'an 223400, Jiangsu Province, China \\ *Corresponding author: Guixia Jiang, 270764968@qq.com
}

\begin{abstract}
Objective: To study the clinical nursing effect of early rehabilitation nursing on stroke patients with hemiplegia in neurology department. Methods: Stroke patients with hemiplegia treated in our hospital from March 2018 to March 2020 were selected for the present study. Twenty patients in each group were divided into two groups by random number table method, either in experimental group or control group. Routine nursing was used in the control group, while early rehabilitation nursing was carried out in the experimental group. The clinical nursing effect of the two groups of patients was compared. Results: After nursing, the neurological function of the patients was significantly improved $(\mathrm{P}<0.05)$, the improvement of the experimental group was better than that of the control group $(\mathrm{P}<0.01)$, the daily activities of the patients in the experimental group were significantly improved, and the score of the experimental group was higher than that of the control group $(\mathrm{P}<0.05)$. After nursing, anxiety and depression scores in the experimental group were significantly lower than those in the control group $(\mathrm{P}<0.05)$, and the recovery of limb function in the experimental group was better than that in the control group $(\mathrm{P}<0.05)$. Conclusion: The implementation of early rehabilitation nursing measures plays an important role in the recovery of stroke patients with hemiplegia by promoting the recovery of neurological function, improving the psychological state of patients, and promoting the recovery of limbs.
\end{abstract}

Keywords: Department of Neurology; Stroke; Hemiplegia; Early rehabilitation nursing

Publication date: November 2021; Online publication: November 30, 2021

\section{Introduction}

The improvement of economic level is accompanied by a huge change to people's living habits and more diversified diet. Alcohol drinking, high intake of fat and salt, and the habit of staying up are some of the examples of the changes that may lead to stroke. The increasing incidence rate of stroke is alarming, and is accompanied by high mortality and disability rates. The elderly population is the high-risk group of the disease. It is also important to note that most stroke patients do not have ideal prognosis. Hemiplegia is the most common sequela of stroke. Patients with physical disorders, such as hemiplegia, are predisposed to adverse emotions such as anxiety and depression, which will have an adverse impact on the subsequent recovery of patients. Therefore, it is necessary to carry out nursing intervention for stroke patients with hemiplegia in order to enhance recovery speed, improve living ability and improve their prognosis ${ }^{[1]}$. The current study selected patients in our hospital from March 2018 to March 2020 to study and analyze the nursing effect of early rehabilitation nursing on stroke patients with hemiplegia.

\section{Materials and methods}

\subsection{General information}

Forty stroke patients with hemiplegia treated in our hospital from March 2018 to March 2020 were selected for the present study. Using the random number table method, the patients were divided into two groups: 
the experimental group $(n=20)$ and the control group $(n=20)$. There were 12 males and 8 females in the

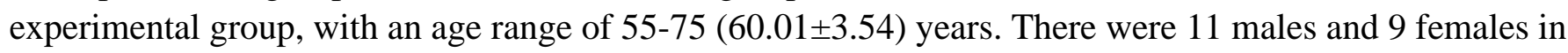
the control group, with an age range of 57-78 (62.14 \pm 3.67$)$ years.

The study participants who were admitted to the study were patients clinically diagnosed with stroke. The subjects were briefed about specific contents of nursing. Patients with the following characteristics were excluded from the study: (1) patients with intracerebral hemorrhage based on brain computed tomography (CT); (2) patients with other serious complications who were unable to participate in nursing research; (3) patients accompanied by mental illness; and (4) patients with previous cerebrovascular diseases and sequelae. There was no significant difference in general data between the two groups $(\mathrm{P}>$ $0.05)$.

\subsection{Methods}

The two groups were given the same treatment measures to control blood pressure, maintain water electrolyte balance, and prevent infection by using antibiotics. The control group was given routine nursing and instructed to take medicine on time. Investigators observed the changes of patients' condition and recorded observations in time, and comforted the patients.

The experimental group received early rehabilitation treatment.

\section{(1) Bed rest care}

Early rehabilitation nursing can effectively reduce the disability rate of patients. This involves massaging the patients, assisting the patient in limb passive exercise (with the aid of family members), preventing edema and pressure ulcer, and avoiding muscle atrophy. The patient should be turned over regularly and in an alternate manner for side lying and flat lying positions while keeping them comfortable. The lying position should be exchanged between the affected side and the healthy side regularly. The affected limb of the patient should be massaged, massaging from the distal end to the proximal end during the swelling period, in order to promote blood circulation through external force. After the swelling of the affected limb is eliminated, centrifugal massage can be performed from the proximal end to the distal end to promote the recovery of the affected limb. It is necessary to help patients with passive exercises such as moving joints, limbs and other parts to promote blood flow ${ }^{[2]}$. Self-turning over nursing exercises involves relying on the patient's ability to turn over independently in bed. The nursing staff guides the patient to practice elbow bending and complete the turning with the help of the normal side. After the normal side turns over skillfully, patients can turn over to the affected side step by step. Under the guidance of nursing staff, the patient is used to turning over with the strength of upper and lower limbs on the normal side. After turning over, nursing staff can help the patient adjust the lying position. If necessary, a cushion should be put under the affected limb to prevent pressure ulcer. Nursing staff should keep the patient in the supine position, keep his body relaxed, guide the patient to carry out upper and lower limb muscle control exercise, and practice upper and lower limb muscle relaxation and tension exercise according to the command of the nursing staff.

\section{(2) Activity and posture nursing}

Patients in the acute stage should stay in bed and the nursing staff should pay attention to maintaining the functional position of paralyzed limbs. Movable denture should be removed from the comatose patients to prevent accidental swallowing and ensure the unobstructed respiratory tract. For patients with unconsciousness, restlessness, temporal lobe infarction and mental symptoms, the use of guardrails and restraint belts should be considered when necessary to prevent falling and hurting people. When the condition is stable, functional exercise of paralyzed limbs is carried out to prevent joint deformity and muscle atrophy. For example, patients turn the head to the affected side, and comb hair with the healthy 
hand from the forehead of the healthy side to the back of the head and neck; this requires the fingers pressing the scalp tightly and slowly pushing back for 20 times. Patients pinch and squeeze the affected hand, place the affected hand in front of the chest, pinch and squeeze it from the distal end to the proximal end along both sides of each finger on the affected side with a healthy thumb and index finger, and press it tightly at the root of the proximal end of the finger for 20 seconds. Patients repeat 5 times for each finger and carry out necessary recovery exercises, such as surrounding face washing, half bridge hip lifting, resistance leg clamping, leg warping and hip swinging. Elderly and hypertensive patients should avoid sudden change of body position, keep the sheets clean, dry and flat, turn over, pat backs and aspirate sputum as needed when necessary.

\section{(3) Psychological rehabilitation nursing}

With the development of medical technology, people attach great importance to patients' mental health while paying attention to disease rehabilitation. For stroke patients with hemiplegia, psychological care is very necessary. Many patients who lost limb control ability are at risk of suffering from anxiety, depression, pessimism, and irritability. The continuous suffering from such adverse emotions will have a negative effect on the recovery of patients, which is not conducive to the later recovery. Therefore, psychological intervention is necessary to comfort and encourage patients, and adjust their mentality so that they actively participate in treatment ${ }^{[4]}$.

(4) Dietary care

The principle of dietary requirements is low salt ( $6 \mathrm{~g} /$ day $)$, low fat, low cholesterol, an appropriate amount of carbohydrates and rich vitamins. The patients should consume less fat, lard, cream, egg yolk, hairtail, animal viscera, candy and sweets, but instead consume more lean meat, fish and shrimp, bean products, fresh vegetables, fruits and iodine-containing food. Taking edible vegetable oil is advocated. It is also necessary to avoid spicy food, quit smoking and alcohol, drink tea, drink more water (2000 ml), eat regularly, and avoid overeating or excessive hunger. Hemiplegic patients should have their meals in the lying position with their normal side. When eating, they should take the sitting position or raise the head of the bed by $30^{\circ}$, and cushion the pillow under the head to bend the head forward. For patients with dysphagia, they should choose to eat in a sitting position and bend their head slightly forward. For patients who can't sit up, they should take the supine position and roll up the head of the bed by $30^{\circ}$. They should choose foods that are convenient for swallowing, i.e., soft, uniformly dense, and appropriately viscous. Empty swallowing, alternating swallowing of food, side swallowing and nodding swallowing can be used to facilitate food into the esophagus. Patients who cannot swallow should be given diet for nasal feeding according to the norms of enteral nutrition.

\subsection{Evaluation criteria}

The degree of neurological deficit before and after treatment was evaluated by the international NIH Stroke Scale/Score (NIHSS), and the ability of daily activities was evaluated by the revised Barthel index. Hamilton Depression Scale (HAMD) and Hamilton Anxiety Scale (HAMA) were used to compare the anxiety and depression of patients before and after nursing, and Fugl-Meyer Assessment Scale (FMA) was used to evaluate limb function.

\subsection{Statistical analysis}

SPSS 24.0 statistical software was used to process the data, and the measurement data are expressed as mean \pm standard deviation $(\mathrm{SD})$ and $\mathrm{t}$ test. $\mathrm{P}<0.05$ indicates that the difference is statistically significant. 


\section{Results}

\subsection{Comparison of NIHSS scores before and after nursing (Table 1)}

Table 1. Comparison of NIHSS scores before and after nursing

\begin{tabular}{lccc}
\hline Group & Before nursing & After nursing for 1 month & After nursing for 3 months \\
\hline Experimental group $(\mathrm{n}=20)$ & $20.12 \pm 3.56$ & $10.45 \pm 3.46$ & $7.46 \pm 2.50$ \\
Control group $(\mathrm{n}=20)$ & $20.14 \pm 3.50$ & $15.64 \pm 2.97$ & $10.24 \pm 2.46$ \\
$\mathrm{t}$ value & 0.018 & 5.090 & 3.545 \\
$\mathrm{P}$ value & 0.986 & 0.000 & 0.001 \\
\hline
\end{tabular}

Data are expressed as mean $\pm \mathrm{SD}$.

\subsection{Comparison of Barthel index before and after treatment (Table 2)}

Table 2. Comparison of Barthel index before and after treatment

\begin{tabular}{lccc}
\hline Group & Before nursing & After nursing for 1 month & After nursing for 3 months \\
\hline Experimental group $(\mathrm{n}=20)$ & $33.54 \pm 3.69$ & $65.74 \pm 3.16$ & $87.37 \pm 2.55$ \\
Control group $(\mathrm{n}=20)$ & $34.76 \pm 3.20$ & $59.13 \pm 3.05$ & $68.41 \pm 2.59$ \\
$\mathrm{t}$ value & 1.117 & 6.731 & 23.329 \\
$\mathrm{P}$ value & 0.271 & 0.000 & 0.000 \\
\hline
\end{tabular}

Data are expressed as mean \pm SD.

\subsection{Comparison of psychological state before and after nursing (Table 3)}

Table 3. Comparison of psychological state before and after nursing

\begin{tabular}{|c|c|c|c|c|}
\hline \multirow[t]{2}{*}{ Group } & \multicolumn{2}{|c|}{ HAMD } & \multicolumn{2}{|c|}{ НАМА } \\
\hline & Before nursing & After nursing & Before nursing & After nursing \\
\hline Experimental group $(n=20)$ & $23.15 \pm 1.44$ & $8.64 \pm 1.25$ & $20.84 \pm 1.12$ & $9.32 \pm 1.75$ \\
\hline Control group $(\mathrm{n}=20)$ & $23.20 \pm 1.48$ & $13.03 \pm 1.62$ & $20.96 \pm 1.46$ & $14.48 \pm 1.33$ \\
\hline $\mathrm{t}$ value & 0.108 & 9.595 & 0.292 & 10.499 \\
\hline$P$ value & 0.914 & 0.000 & 0.772 & 0.000 \\
\hline
\end{tabular}

Data are expressed as mean \pm SD.

\subsection{Comparison of limb function recovery before and after nursing (Table 4)}

Table 4. Comparison of limb function recovery before and after nursing

\begin{tabular}{|c|c|c|c|c|}
\hline \multirow[t]{2}{*}{ Group } & \multicolumn{2}{|c|}{ FMA } & \multicolumn{2}{|c|}{ FAC } \\
\hline & Before nursing & After nursing & Before nursing & After nursing \\
\hline Experimental group $(\mathrm{n}=20)$ & $10.54 \pm 1.64$ & $28.64 \pm 1.41$ & $2.84 \pm 0.12$ & $9.55 \pm 1.25$ \\
\hline Control group $(\mathrm{n}=20)$ & $11.20 \pm 1.38$ & $23.03 \pm 1.57$ & $2.96 \pm 0.76$ & $6.48 \pm 1.46$ \\
\hline t value & 1.377 & 11.889 & 0.697 & 7.143 \\
\hline$P$ value & 0.177 & 0.000 & 0.490 & 0.000 \\
\hline
\end{tabular}

Data are expressed as mean \pm SD. 


\section{Discussion}

Stroke is a common cardiovascular and cerebrovascular disease. It is accompanied by acute onset, and subsequently rapid disease progression and the development of sequelae. Stroke patients with hemiplegia often have motor neuron and central nerve injury. After drug treatment, a long period is needed for recovery. If the necessary nursing is not provided, the prognosis of patients is often not ideal. Early rehabilitation nursing for stroke patients with hemiplegia can help patients with muscle and joint training as soon as possible, promote the recovery of motor function and improve the prognosis of patients ${ }^{[5]}$.

In this study, the neurological function of the two groups recovered after nursing. The NIHSS score of the experimental group was lower than that of the control group $(\mathrm{P}<0.05)$, and the daily activity ability of the patients was significantly improved $(\mathrm{P}<0.05)$. Compared with the control group, the Barthel index of the experimental group was higher, and the difference was statistically significant $(\mathrm{P}<0.05)$. After analysis, we found that the HAMD and HAMA scores of the experimental group were significantly lower than those of the control group $(\mathrm{P}<0.05)$. After nursing, the limb recovery of the experimental group was better than that of the control group $(\mathrm{P}<0.05)$. This shows that early rehabilitation nursing can promote the recovery of neurological function. After nursing, the ability of daily living of patients is significantly improved, and the recovery of limb function is significant. Early rehabilitation nursing can prevent and reduce joint contracture, improve muscle tension, and avoid all kinds of misuse syndrome and disuse syndrome. Psychological nursing can adjust emotions in a positive manner as well as eliminate anxiety, depression and other bad emotions, fostering a positive attitude in patients while receiving treatment . Early rehabilitation nursing can also improve motor function and self-care ability, prevent disability, effectively improve the quality of life of patients with hemiplegia, reduce the burden of family and society, and has good clinical application value.

\section{Disclosure statement}

The author declares no conflict of interest.

\section{References}

[1] Sun H, Xu J, 2021, Effect of Early Comprehensive Rehabilitation Nursing on Limb Movement and Nerve Function in Patients with Hemiplegia after Stroke. Guizhou Medical Journal, 45(2): 330-331.

[2] Song Y, 2021, Effect of Early Rehabilitation Nursing Intervention on Prognosis of Hemiplegia after Ischemic Stroke. Chinese Practical Medicine, 16(8): 179-181.

[3] Xie H, Yang CH, 2021, Clinical Application of Early Rehabilitation Nursing Pathway in Cerebral Apoplexy Patients with Hemiplegia. China Health Standard Management, 12(3): 140-142.

[4] Wu WW, 2021, Effect of Early Comprehensive Rehabilitation Nursing on Limb Function and Quality of Life in Patients with Hemiplegia after Stroke. Journal of Contemporary Clinical Medicine, 34(1): 24,8 .

[5] Liu LL, 2021, Effect of Early Rehabilitation Nursing Intervention on Limb Function and Quality of Life in Patients with Hemiplegia after Stroke. Guide of China Medicine, 19(9): 208-209. 